White Paper for U.S. Army Rapid

Equipping Force: Waste Heat Recovery with Thermoelectric and Lithium-Ion Hybrid Power System

J. C. Farmer

November 27, 2007 
This document was prepared as an account of work sponsored by an agency of the United States government. Neither the United States government nor Lawrence Livermore National Security, LLC, nor any of their employees makes any warranty, expressed or implied, or assumes any legal liability or responsibility for the accuracy, completeness, or usefulness of any information, apparatus, product, or process disclosed, or represents that its use would not infringe privately owned rights. Reference herein to any specific commercial product, process, or service by trade name, trademark, manufacturer, or otherwise does not necessarily constitute or imply its endorsement, recommendation, or favoring by the United States government or Lawrence Livermore National Security, LLC. The views and opinions of authors expressed herein do not necessarily state or reflect those of the United States government or Lawrence Livermore National Security, LLC, and shall not be used for advertising or product endorsement purposes.

This work performed under the auspices of the U.S. Department of Energy by Lawrence Livermore National Laboratory under Contract DE-AC52-07NA27344. 


\section{White Paper for U.S. Army Rapid Equipping Force: Waste Heat Recovery with Thermoelectric and Lithium-Ion Hybrid Power System}

\section{Prepared for:}

Wade Jost

Science and Technology Advisor

Ideal Innovations Incorporated

U.S. Army Rapid Equipping Force

10236 Burbeck Road

Fort Belvoir, Virginia 22060-5852

Mobile (253) 509-2013

Email:Wade.jost@us.army.mil

Email: Wade.jost@sbelvoirdms.army.smil.mil

Web Site: https://www.ref.army.mil/

Prepared by:

Dr. Joseph Farmer

CMELS Directorate Senior Scientist

Chemistry, Materials, Environmental \& Life Sciences (CMELS) Directorate

Associate Program Leader for Materials Research

Energy Program, Global Security Directorate

Lawrence Livermore National Laboratory

7000 East Avenue / PO Box 808

Livermore, California 94550

Telephone: 925-423-6574

Mobile: 925-337-1188

Email: farmer4@,lnl.gov

\section{Abstract}

By harvesting waste heat from engine exhaust and storing it in light-weight high-capacity modules, it is believed that the need for energy transport by convoys can be lowered significantly. By storing this power during operation, substantial electrical power can be provided during long periods of silent operation, while the engines are not operating. It is proposed to investigate the potential of installing efficient thermoelectric generators on the exhaust systems of trucks and other vehicles to generate electrical power from the waste heat contained in the exhaust and to store that power in advanced power packs comprised of polymergel lithium ion batteries. Efficient inexpensive methods for production of the thermoelectric generator are also proposed. The technology that exists at LLNL, as well as that which exists at industrial partners, all have high technology readiness level (TRL). Work is needed for integration and deployment. 


\section{Concept}

Statistics quoted by various Federal agencies indicate that seventy-percent of the convoys in Iraq involve the transportation of fuel, battery and water (energy and water) for our troops. Ultimately, any increase in fuel efficiency, or any ability to harvest waste heat and store the associated energy should help lower the number of convoys required to supply energy needs.

By harvesting waste heat from engine exhaust and storing it in light-weight high-capacity modules, it is believed that the need for energy transport can be lowered significantly.

Specifically, it is proposed to investigate the potential of installing efficient thermoelectric generators on the exhaust systems of trucks and other vehicles to generate electrical power from the waste heat contained in the exhaust, and to store that power in advanced power packs comprised of polymer-gel lithium ion batteries. This novel hybrid energy concept is illustrated with Figure 1.

As will be discussed in the following section, a variety of thermoelectric materials exist for the construction of a generator. Despite theoretical gains in the efficiency (dimensionless figure-ofmerit) of thermoelectric devices that may eventually be possible with $2 \mathrm{D}$ quantum well films with enhanced carrier transport, or multilayer superlattices that achieve improvements in efficiency through enhanced phonon scattering, it is believed that the most realistic choices for waste heat recovery from an exhaust system remain generators made from p-type $\mathrm{Bi}_{0.5} \mathrm{Sb}_{1.5} \mathrm{Te}_{3}$ $(\mathrm{ZT} \sim 0.98)$ and n-type $\mathrm{Bi}_{2} \mathrm{Te}_{1.4} \mathrm{Se}_{0.6}(\mathrm{ZT} \sim 0.72)$. Devices can also be constructed from other materials, such as doped $\mathrm{PbTe}(\mathrm{ZT} \sim 1.05)$.

While discrete elements of these of bulk semiconductors are usually fabricated as discrete elements with conentional powder metallurgy techniques, such as pressing and sintereing, it may now be posible to fabricate generators by direct deposition of the n-type and p-type materials onto a cylindrical tube that can be incorporated into the exhaust system. Since the coatings must be several millimeters thick to work efficiently, the deposition meted of choice will probably be cold-spray, though other techniques also exist.

The secondary battery system proposed for this application will be constructed from individual polymer-gel lithium-ion batteries. The best commercially available, polymer-gel lithium ion battery now has a specific energy of $\sim 180 \mathrm{Wh} / \mathrm{kg}$, an energy density of $\sim 350 \mathrm{Wh} / \mathrm{L}$, and a reasonably good rate capability, allowing discharge at $\mathrm{C} / 2$. 
White Paper for U.S. Army Rapid Equipping Force - Joseph C. Farmer November $22^{\text {nd }} 2007$

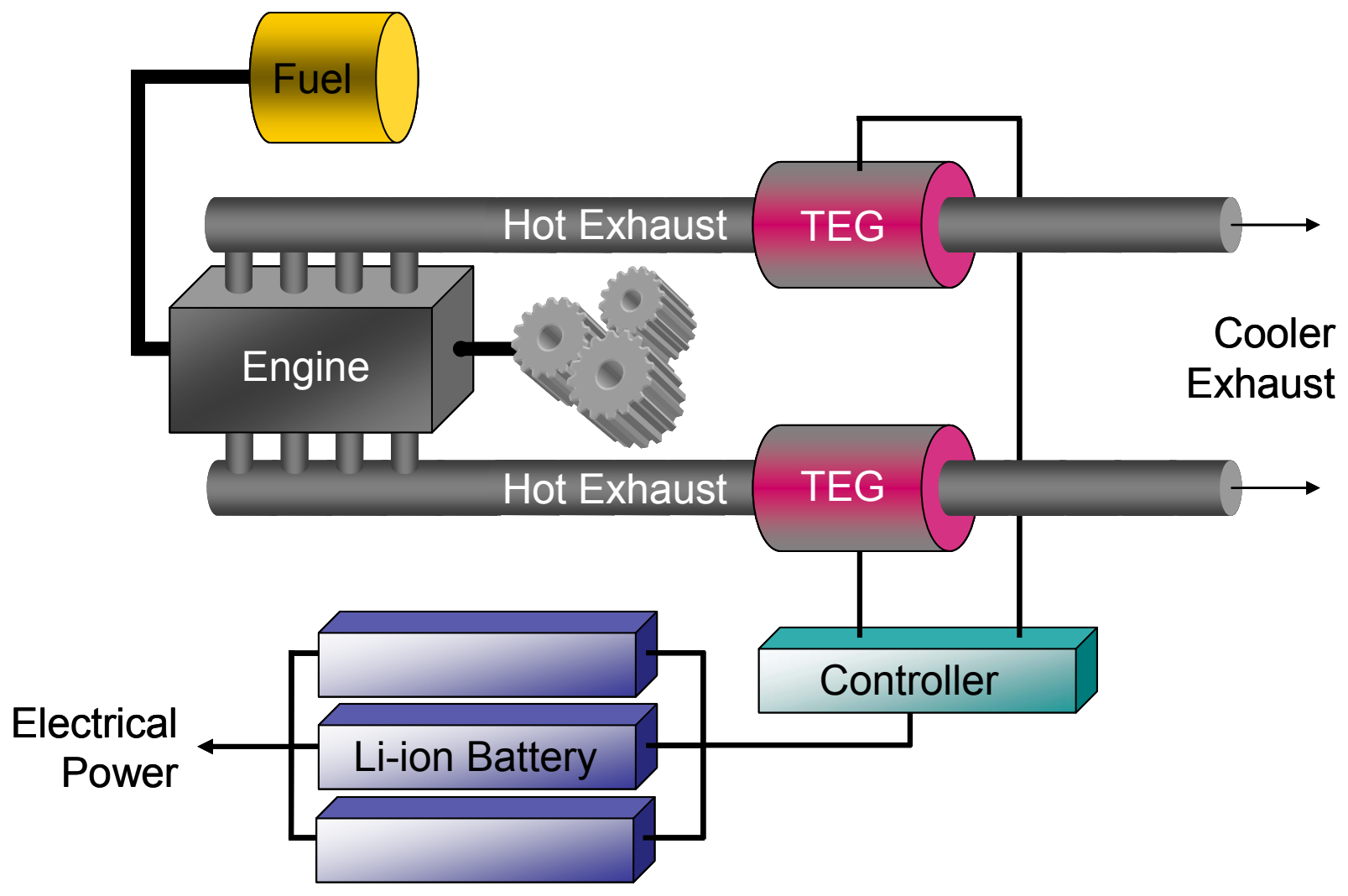

Figure 1 - Hybrid power system for recovery of waste heat from exhaust systems, using thermoelectric generation coupled with high-capacity light-weight lithium ion batteries. 


\section{Supporting Information}

\section{Solid-State Thermoelectric Power Generation}

Solid-state thermoelectric generators have no moving parts, and can be used for the reliable direct conversion of heat to electrical energy, with exceptional reliability in remote and inaccessible locations, including deep space. Such energy converters could be powered on the sea floor in a variety of ways, including geothermal heat sources, heat from the combustion of natural gas from deep ocean wells, decay heat from radioisotopes, and small deployable nuclear reactors. Examples of thermoelectric generators that have been powered by small nuclear reactors and radioisotope sources include:

- SNAP 10: Reactor-Powered Thermoelectric Generator

- SNAP 19: Radioisotope Thermoelectric Generator

- SNAP 27: Radioisotope Thermoelectric Generator

Radioisotope sources with the necessary power density for the applications of interest to the oil and gas industry are believed to be too limited for serious consideration, but are included for completeness. Small deployable proliferation-resistant reactors may become a viable option in the coming years, and are now being considered for other applications.

Thermoelectric power generators are $p-n$ junctions in which charge carriers and heat flow in parallel. Electrons and holes must acquire energy at the p-n junction to flow in a direction opposite to the temperature gradient (from cold to hot). Both ohmic heating and heat conduction must be minimized for the efficient operation of such devices. The thermodynamic efficiency of a thermoelectric power generator $(\eta)$ is calculated from the dimensionless figure of merit (ZT). The dimensionless figure of merit, ZT, is determined by Seebeck coefficient $(\alpha)$, electrical conductivity $(\sigma)$, electronic thermal conductivity $\left(\kappa_{\mathrm{el}}\right)$, and lattice thermal conductivity $\left(\kappa_{\mathrm{ph}}\right)$.

$$
Z T=\frac{\sigma \alpha^{2}}{\kappa_{p h}+\kappa_{e l}} T
$$

The expressions for $\eta$ at the optimum current level is:

$$
\eta=\frac{T_{h}-T_{c}}{T_{h}} \frac{\sqrt{1+Z T}-1}{\sqrt{1+Z T}+T_{c} / T_{h}}
$$

where $T$ is the average temperature of the device, $T_{h}$ is the hot temperature and $T_{c}$ is the cold temperature [Angrist, 1976]. To achieve high values of $\eta$ with a thermoelectric device, a material with a large ZT value must be found [Vining, 1992]. The dependence of $\eta$ on ZT, $\mathrm{T}_{\mathrm{c}}$, and $T_{h}$ is illustrated by Figure 2 . 
White Paper for U.S. Army Rapid Equipping Force - Joseph C. Farmer November $22^{\text {nd }} 2007$

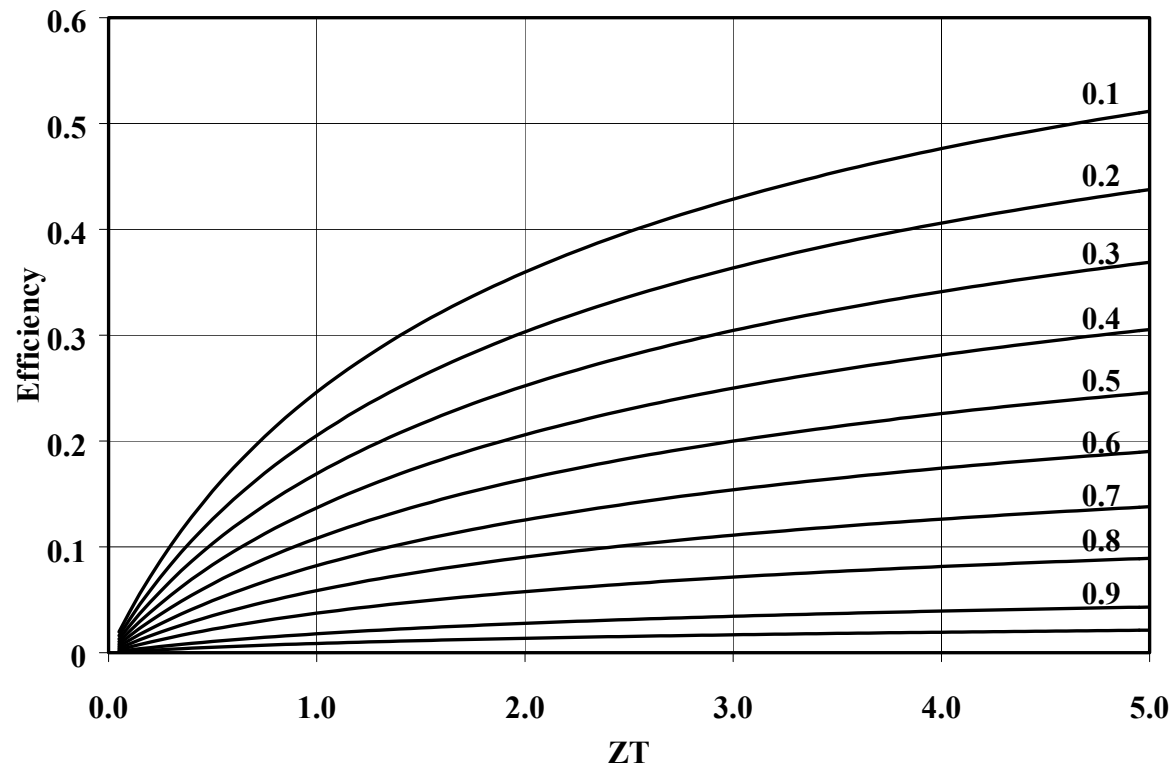

Figure 2 - Thermodynamic efficiency $(\eta)$ as a function of $Z T$ for $T_{c} / T_{h}$ ratios from 0.1 to 0.9 .

The efficiency of thermoelectric generators is limited by the properties of the solid state materials used in their construction, and the available temperature gradient. Promising thermoelectric materials should have high "power factors" $\left(\sigma \alpha^{2}\right)$ and low thermal conductivities $\left(\kappa_{\mathrm{ph}}+\kappa_{\mathrm{el}}\right)$. Furthermore, such materials should be plentiful enough (and sufficiently inexpensive) to enable the possible construction of large-scale devices. Degenerate semiconductors have the best combinations of these intrinsic properties [Cornish, 1959]. Strategies to find bulk thermoelectric materials with exceptional figures of merit require that we simultaneously increase $\sigma$ and $\alpha$, while decreasing $\kappa_{\mathrm{ph}}+\kappa_{\mathrm{el}}$. Unfortunately, improvements in $\sigma$ are accompanied by detrimental changes in $\kappa_{\mathrm{el}}$ since $\sigma$ and $\kappa_{\mathrm{el}}$ trend in the same direction (Wiedemann-Franz law). Since $\alpha, \sigma$, and $\kappa_{\mathrm{el}}$ are all sensitive to carrier concentration, ZT is optimized by changing the extent of doping. Overall increases in the optimum value of ZT are achieved by reducing $\kappa_{\mathrm{ph}}$ by other means. For example, semiconductor compounds made from elements with high atomic mass tend to have lower lattice thermal conductivities than those made from lighter elements. This is illustrated by Keyes' rule and similar expressions which correlate lattice thermal conductivity $\left(\kappa_{\mathrm{ph}}\right)$ with melting-point temperature $\left(T_{m}\right)$, atomic mass $(A)$, and density $(\rho)$.

$$
\eta=\frac{B T_{m}^{3 / 2} \rho^{2 / 3}}{A^{7 / 6} T}
$$

where $\mathrm{B}$ is a constant and $\mathrm{T}$ is the temperature. It is evident that semiconductors with relatively low melting points should be used for low-temperature applications; i.e., as $\mathrm{T}$ is reduced, $\mathrm{T}_{\mathrm{m}}$ must be reduced to compensate. Furthermore, it is clear that materials made of elements with high atomic masses are preferred.

Bismuth Telluride. Materials favored for low-temperature applications include: PbTe; 25 percent p-type $\mathrm{Bi}_{2} \mathrm{Te}_{3}$ and 75 percent $\mathrm{Sb}_{2} \mathrm{Te}_{3}$ with $1.75 \%$ excess Te; and n-type 75 percent $\mathrm{Bi}_{2} \mathrm{Te}_{3}$ and 25 
percent $\mathrm{Bi}_{2} \mathrm{Se}_{3}$. The general class of compounds represented by $\mathrm{Bi}_{2 x} \mathrm{Sb}_{2(1-x)} \mathrm{Te}_{3 y} \mathrm{Se}_{(3-\mathrm{y})}$ are rhombohedral and are classified as V-VI narrow-band $(\leq 0.2 \mathrm{eV})$ semiconductors. These materials are stable at low temperatures, but disproportionate above $600 \mathrm{~K}$. Dimensionless figures of merit (ZT's) for these materials have been published and are tabulated in Table 3 [Wood, 1988; Rosi, 1968]. The unusually low thermal conductivities of these bismuthcontaining materials, which are less than $10 \mathrm{~mW} \mathrm{~cm}^{-1} \mathrm{~K}^{-1}$ at $300 \mathrm{~K}$, are due to the formation of a solid solution between isoelectronic, heavy-element, compound semiconductors. Such alloying is believed to lead to more intense scattering of phonons than electrons, thereby decreasing $\kappa_{\mathrm{ph}}$ without reducing $\sigma$ significantly [Wood, 1988; Ioffe, 1956]. The phonons responsible for heat conduction have wavelengths equivalent to a few interatomic spacings. Since the conduction of these phonons involves atomic motions, they are scattered most effectively by atomic-scale mass variations in the lattice. Fortunately, the scattering of charge carriers by such variations is minimal. If atoms of higher mass but similar valence and ionic size are substituted into a lattice, the effect on the periodic electrostatic potential of the lattice should be small. It has also been suggested that electrons in semiconducting compounds travel on the cation (metal atom) sublattice while holes move on the anion (non-metal atom) sublattice [Hyun, 1992; Wood, 1988; Airapetiants, 1957]. Thus, defects in the cation sublattice will scatter electrons more strongly than defects in the anion sublattice. Furthermore, defects in the anion sublattice will scatter holes more strongly than defects in the cation sublattice. Therefore, in the case of Group V chalcogenides, the addition of $\mathrm{Bi}_{2} \mathrm{Se}_{3}$ to $\mathrm{Bi}_{2} \mathrm{Te}_{3}$ should produce a larger increase in the n-type figure of merit than the addition of $\mathrm{Sb}_{2} \mathrm{Te}_{3}$. The latter should produce better p-type material. A detailed model has been developed to predict the thermoelectric properties of this alloy system as a function of composition [Fleurial, 1992]. Several optical studies of this alloy system have also been done [Wakaki, 1992; Stordeur, 1992].

Silicon Germanium. The best known material for high-temperature TEG's is $\mathrm{Si}_{70} \mathrm{Ge}_{30}$ alloy [Cook, 1992; Slack, 1991; Vining, 1991; Wood, 1988; Rosi, 1968]. Usually, B is used as a dopant to produce p-type $\mathrm{Si}_{70} \mathrm{Ge}_{30}$. A combination of $\mathrm{P}$ and $\mathrm{GaP}$ can be used to produce n-type SiGe. Si, Ge, and Si-Ge alloys have the well-known diamond structure. Dimensionless figures of merit (ZT's) for these materials have also been published and are tabulated in Table 3.

Sulfides. Published data for several transition metal and rare earth sulfides indicate that these materials are also good thermoelectric materials. Cutler and Leavy [1963-64] have investigated nonstoichiometric p-type $\mathrm{Ce}_{(3-\mathrm{x})} \mathrm{S}_{4}$. More recently, Kamarzin et al. [1981] and Gschneidner et al. [1982-88] have investigated n-type $\mathrm{La}_{(3-\mathrm{x})} \mathrm{S}_{4}$. In addition to samples made of pressed and sintered powders, single crystals have been studied. These materials are ionic compounds and maintain their bcc structure over the entire range of stoichiometries investigated. Unfortunately, oxysulfides form at high temperature, giving rise to problems with phase stability. Representative data are tabulated in Table 3.

Silicides. We believe that it may be possible to achieve good performance at high temperature with pseudo-binary alloys of (Fe,Os) $\mathrm{Si}_{2}$. Studies have been conducted and published on several silicides, including doped $\mathrm{FeSi}_{2}, \mathrm{Cr}_{28} \mathrm{Si}_{72},\left(\mathrm{CrSi}_{2}\right)_{\mathrm{x}}\left(\mathrm{Cr}_{11} \mathrm{Ge}_{19}\right)_{1-\mathrm{x}}$, and $\mathrm{Ru}_{2} \mathrm{Si}_{3}$ [Birkholz, 1992; Matsubara, 1992; Gladun, 1992; Caillat, 1992; Ohta, 1992]. As shown in Table 3, the thermoelectric properties of these specific materials are poor. However, more recently, theoretical studies [van Ek, 1996; Turchi, 1998] have shown that alloying effects could be used 
to improve the thermoelectric properties of $\mathrm{FeSi}_{2}$. Recent developments in theoretical predictions of alloy stability and transport properties [Turchi, 1995, 1997, 1998; Mayou, 1998] will allow us to improve the performance at high temperature of pseudo-binary alloys such as $(\mathrm{Fe}, \mathrm{Os}, \mathrm{Ru}) \mathrm{Si}_{2}$. The same theoretical technology which is based on a real-space electronic structure description of multi-component alloys, will be also applicable to other geometries such as thin films and quantum wells.

Antimonides. Covalent $\mathrm{IrSb}_{3}$ has a cubic structure and a measured ZT of 0.50 at $773 \mathrm{~K}$ [Caillat, 1992]. Filled skutterudites are an interesting model system to study the decoupling of electrical and thermal transport via structural chemistry [Stacy and Sands, 2002]. The skutterudite structure includes a framework of tilted corner-sharing $\mathrm{CoSb}_{6}$ octahedra giving rise to a narrow-gap semiconductor with moderate thermoelectric properties. Between the corner sharing octahedra are large icosahedral cavities, which may be filled with metal atoms. Limited bonding between this atom and its surrounding cage as well as a mismatch between the atom radius and the size of the cage lead to a large anharmonic thermal parameter for the filling atom; this has been implicated in the significant increases in ZT for filled skutterudites. Finally, most technologically interesting ternary filled skutterudites are very difficult to prepare as anything other than powders; the full characterization of "filling" has been lacking, especially in the ytterbium filled skuterudites. The growth of epitaxial filled skutterudite films potentially opens new analysis routes for studying the filled atom's environment. In the proposed research, the thermoelectric power generation properties of filled skutterudite multilayers will be examined.

Two-Dimensional Quantum Wells. No dramatic improvement in the dimensionless figure of merit, i.e. beyond ZT $>>1$, had been achieved during the thirty years following the explosion in thermoelectrics research experience in the early 1960's. During a rebirth in interest in thermoelectric materials, Hicks and Dresselhaus of MIT have developed a model to predict the thermoelectric properties of two-dimensional quantum wells (2D QWs), which led to several promising predictions [1992-93]. They derived analytical expressions for the intrinsic properties of such quantum wells, including $\alpha, \sigma, \kappa_{\mathrm{el}}$ and $\mathrm{Z}$. Their relatively simple early model assumes that electrons occupy only the lowest sub-band of the quantum well, which is consistent with the optimization of $\mathrm{Z}$ arising from a one-band model, and that there is no quantum-mechanical tunneling through the wide-gap semiconductor. It is assumed that the wide-gap semiconductor does not contribute to the electronic conduction since its carriers occupy lower-energy states in the narrow-gap semiconductor. As described by Dresselhaus, Harmon has used molecular beam epitaxy at MIT Lincoln Laboratory to produce $\mathrm{PbTe} / \mathrm{Te}$ superlattices that appear to have $\mathrm{ZT}_{3 \mathrm{D}} \sim$ 1.9. In these two-dimensional quantum wells, it is believed that both the barrier and quantum well layers are contributing to the figure of merit. The quantum well and barrier layers have about the same thickness. In early theoretical predictions, the contributions of the barrier layer were not accounted for. More recently, Dresselhaus et al. have extended their model to account for partial confinement of carriers in the quantum well. In addition to the effects of quantum well confinement, other factors may also be responsible for the unusually high figure of merit found with these two-dimensional structures. For example, phonon scattering from the interfaces between layers would lower thermal conductivity, and thereby enhance the figure of merit. 
Samples of data are presented here to demonstrate the Team's ability to deliver advanced cutting-edge technology in an area relevant to the proposal. The possibility of creating a twodimensional quantum-well (2D QW) with greatly enhanced dimensionless figures of merit (ZT) has also been explored by the author of this proposal through the actual synthesis of such structures with multi-magnetron sputtering (MMS), as shown in Figure 2 [Farmer et al. 199496]. Several multilayer thermoelectric thin films were synthesized via multi-magnetron sputtering by LLNL, as shown in Figure 3. It was found that sputtering could be used to grow epitaxial single-layer and multilayer films by carefully controlling the composition of the sputtering target, the substrate bias and temperature, the sputtering gas pressure, and the substrate structure. These materials included, but were not limited to:

- $\mathrm{Bi}_{2} \mathrm{Te}_{3} / \mathrm{B}_{4} \mathrm{C} ; \mathrm{Si}_{0.2} \mathrm{Ge}_{0.8} / \mathrm{Si}$

- $\mathrm{Bi}_{0.9} \mathrm{Sb}_{0.1} / \mathrm{PbTe}_{0.8} \mathrm{Se}_{0.2} ; \mathrm{Bi}_{0.9} \mathrm{Sb}_{0.1} / \mathrm{Bi}_{2} \mathrm{Te}_{3} ; \mathrm{Bi} / \mathrm{Bi}_{0.86} \mathrm{Sb}_{0.14}$

- $\left(\mathrm{Bi}_{0.25} \mathrm{Sb}_{0.75}\right)_{2} \mathrm{Te}_{3} /\left(\mathrm{Bi}_{0.25} \mathrm{Sb}_{0.75}\right)_{2}\left(\mathrm{Te}_{0.5} \mathrm{Se}_{0.5}\right)_{3}$.

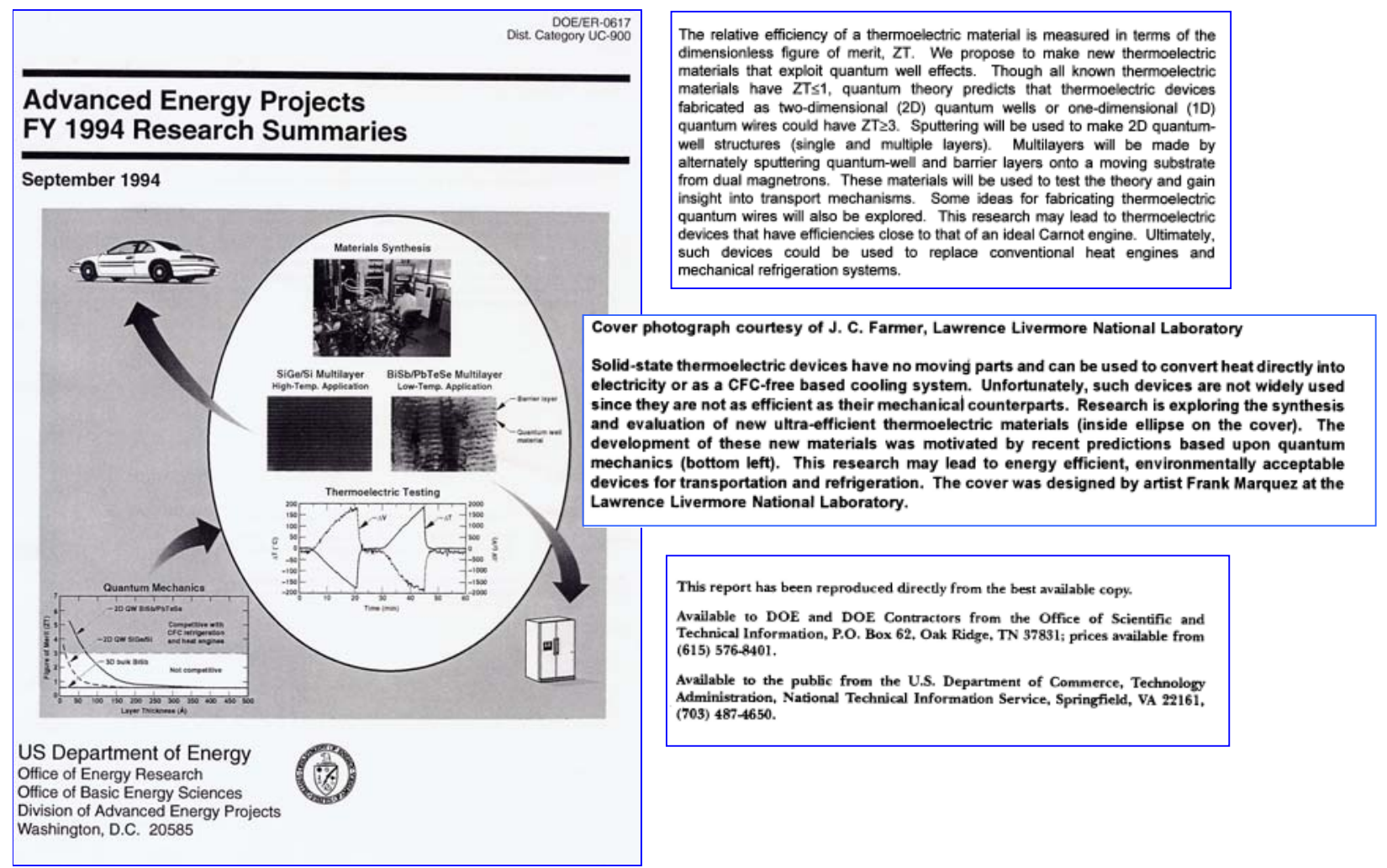

Figure 2 - During the early 1990's, LLNL explored the use of multi-magnetron sputtering to synthesize a wide variety of thermoelectric thin films and devices. 


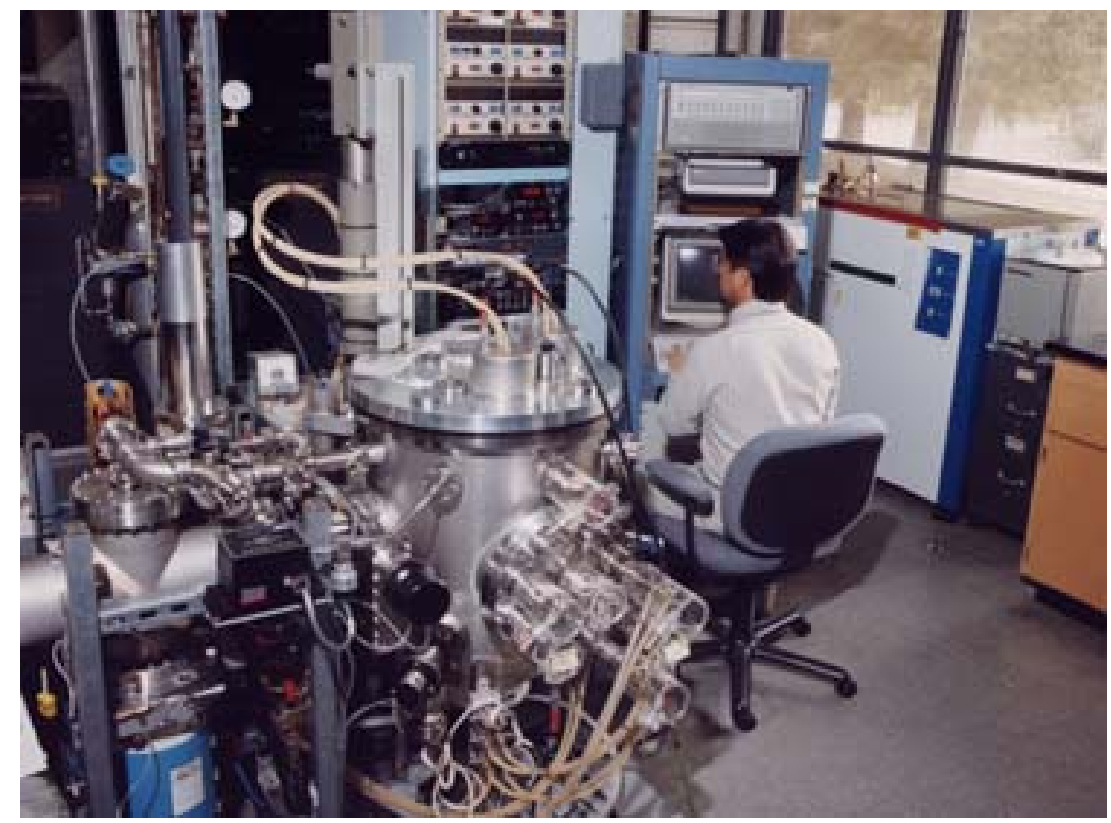

Figure 3 - Multi-magnetron sputter deposition is a practical method for the synthesis of thermoelectric multiplayer films (low dimensional thermoelectric materials).

These films were characterized electronically and structurally. Structural characterization included transmission electron microscopy (Figure 4), X-ray diffraction (Figure 5), and electronic property measurements. In addition to making multilayer films, thin film TE devices were also made and evaluated (Figure 6). It is believed that such process technology would be required for practical fabrication of economically-viable devices. While these devices were not found to have average bulk properties as great as anticipated from theoretical predictions, the program developed synthesis processes capable of producing thermoelectric thin films of the best quality known. In some cases, these films appeared to have figures of merit approaching those of bulk single crystals. This work was pursued to the point where miniature Peltier coolers were fabricated from the thermoelectric thin films.
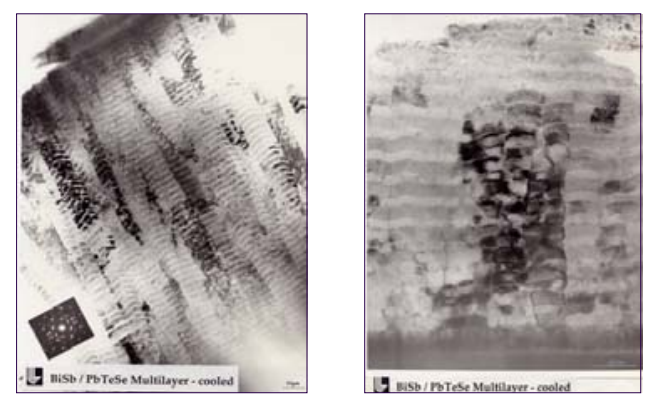

Deposition on cooled substrates

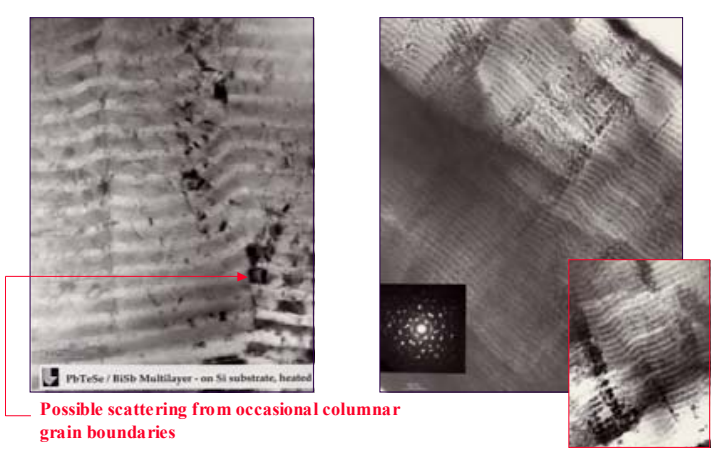

Deposition on heated substrates

Figure 4 - Multi-magnetron sputter deposition has been used to synthesize $\mathrm{Bi}_{0.9} \mathrm{Sb}_{0.1} / \mathrm{PbTe}_{0.8} \mathrm{Se}_{0.2}$ multilayer films with periods as small as $250 \AA$. 
White Paper for U.S. Army Rapid Equipping Force - Joseph C. Farmer November $22^{\text {nd }} 2007$

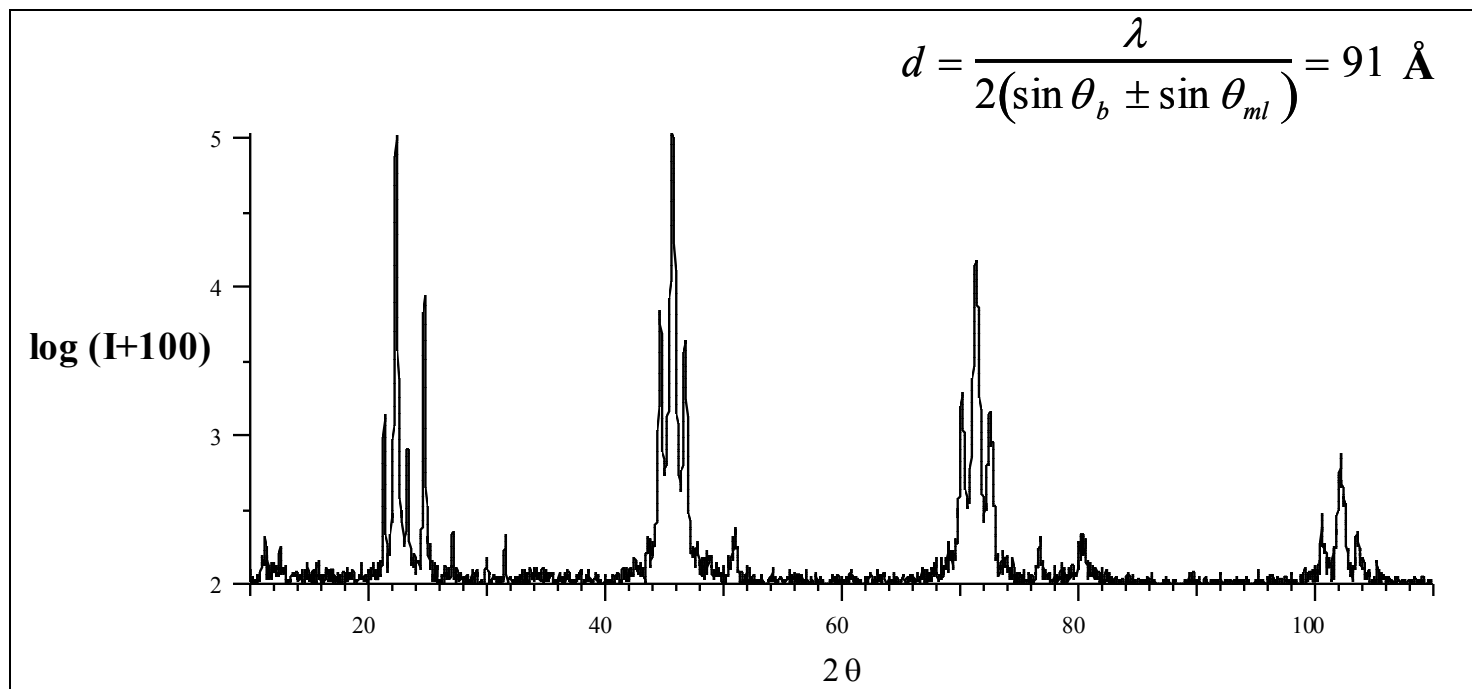

Well-defined satellite peaks evident of epitaxial growth.

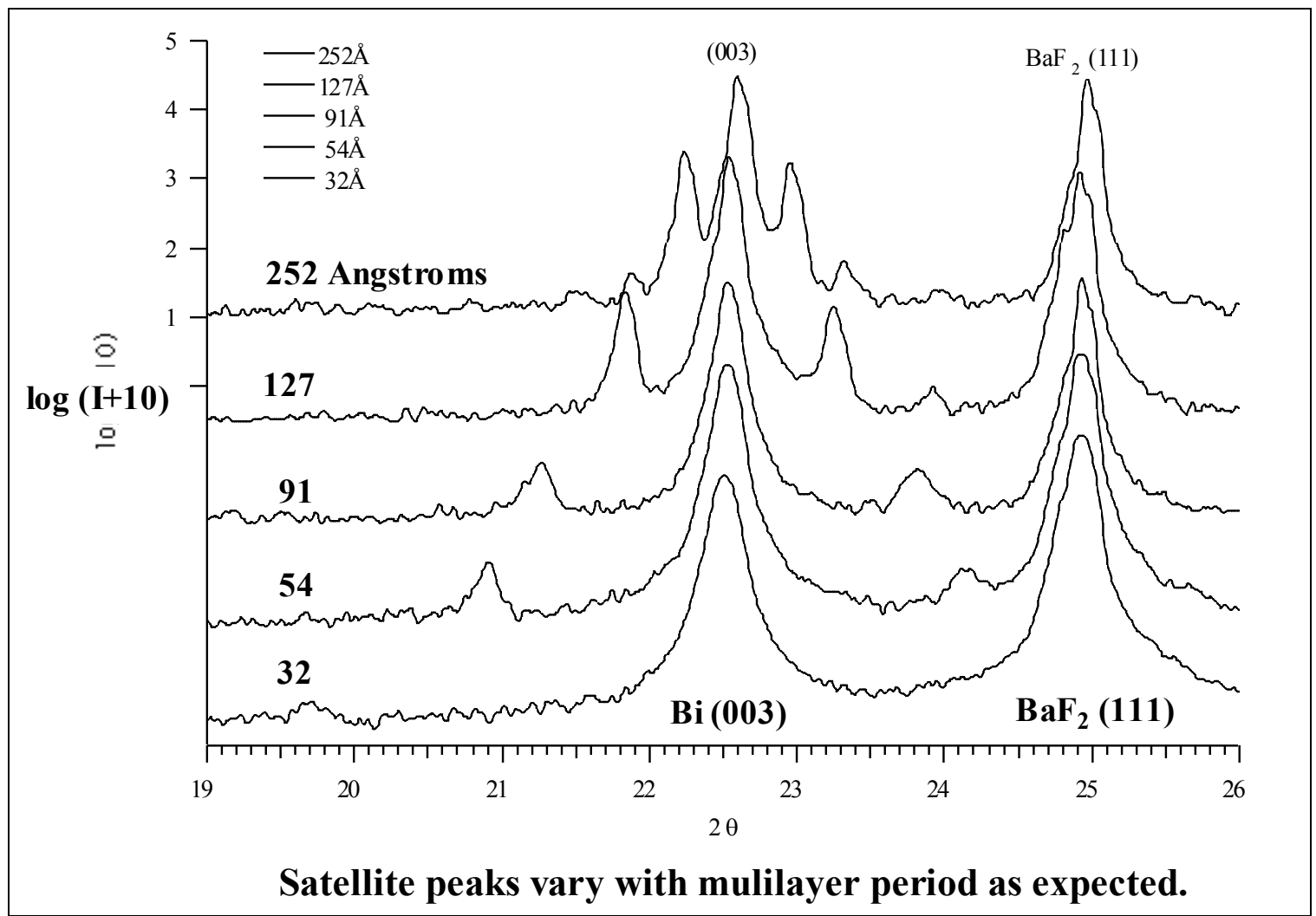

Figure 5 - X-ray diffraction of $\mathrm{Bi} / \mathrm{Bi}_{0.86} \mathrm{Sb}_{0.14}$ thermoelectric multilayer films on $\mathrm{BaF}_{2}$ singlecrystal substrates, thereby demonstrating the possibility of epitaxial grown with sputtering. 
White Paper for U.S. Army Rapid Equipping Force - Joseph C. Farmer November $22^{\text {nd }} 2007$
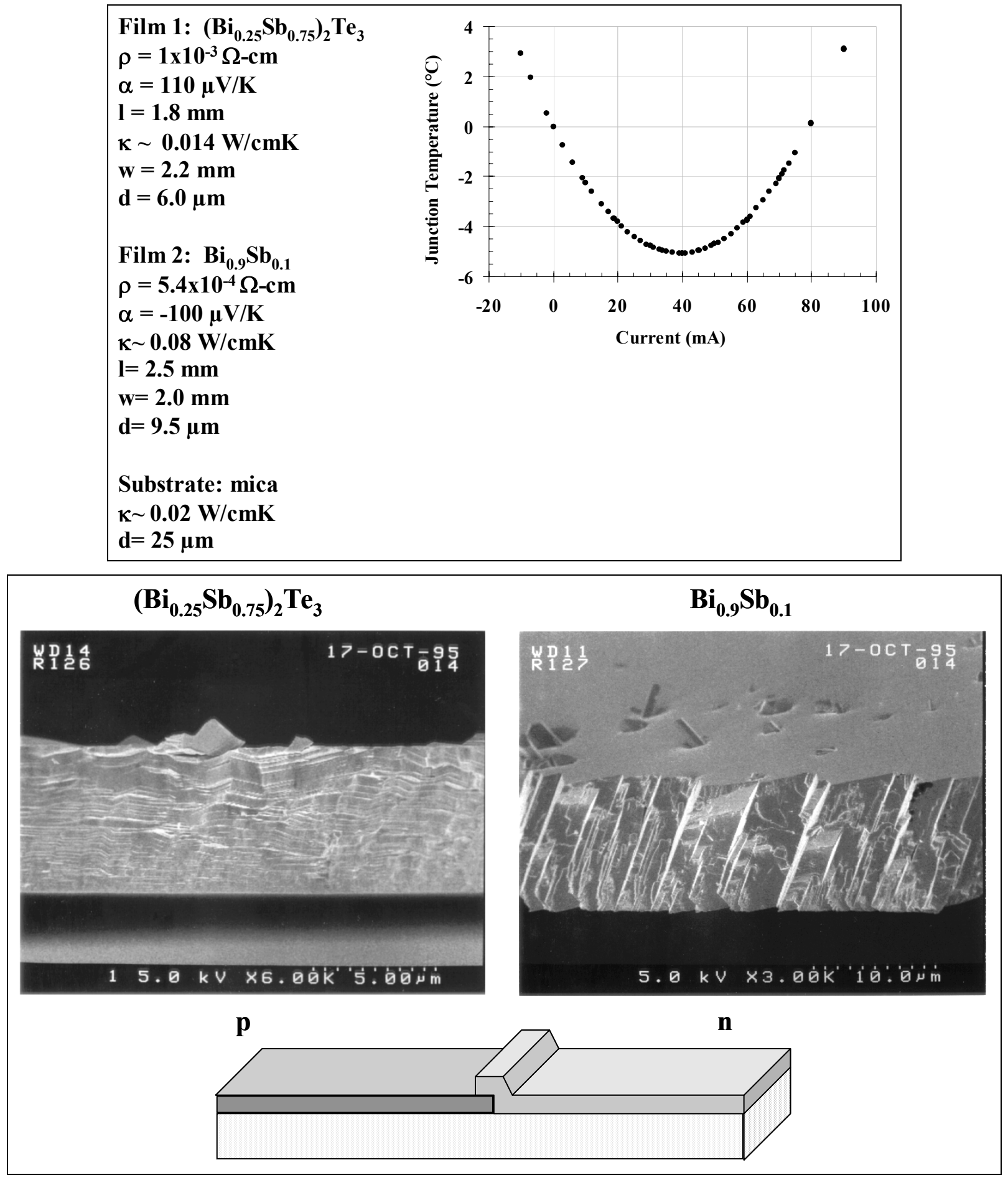

Figure 6 - Miniature thermoelectric device based on sputtered films with p-Type $\left(\mathrm{Bi}_{0.25} \mathrm{Sb}_{0.75}\right)_{2} \mathrm{Te}_{3}$ and $\mathrm{n}-\mathrm{Type} \mathrm{Bi}_{0.9} \mathrm{Sb}_{0.1}$. 
Multilayer Films with Enhanced Interfacial Phonon Scattering. More recently, researchers at Research Triangle Institute (RTI) have synthesized such multilayers, but have exploited transport properties normal to the layers. Interfaces between individual layers scatter phonons, thereby lowering the thermal conductivity. In cases where the differences in electronic properties of individual layers are small, the interfaces contribute little to carrier scattering, and therefore have little detrimental effect on the electronic conductivity, or the Seebeck coefficient. Thus, the dimensionless figure of merit is enhanced. This arrangement lends itself to the skutterudite system of materials, which have very good power factors (electronic properties) but are limited by relatively large thermal conductivities. Structurally "filling" the skutterudites leads to a large reduction in thermal conductivity; several of the filled skutterudites display large figures of merit, in the bulk, at high temperatures. Interfacial phonon scattering in multilayers may further improve the thermal conductivity of these materials. Interdiffusion between layers of variously filled skutterudites avoids the deleterious effects on carrier transport observed in other multilayer systems. Layering filled with unfilled or compositional variation of filled skutterudites may result in a dramatic increase in power generation device performance.

Other Thermoelectric Thin Films. George and Pradeep [1985] and Charles et al. [1988] have prepared films of $\mathrm{Bi}_{2} \mathrm{Te}_{3}$ with excellent thermoelectric properties by reactive coevaporation, achieving stoichiometric composition through careful substrate temperature control. Boikov et al. [1989] reported stoichiometric films of the ternary compound, $\mathrm{Bi}_{0.5} \mathrm{Sb}_{1.5} \mathrm{Te}_{3}$ with thermoelectric properties approaching those of bulk single crystals using flash evaporation. Stolzer and Stordeur [1992] have used planar magnetrons to deposit single-layer $\mathrm{Bi}_{0.5} \mathrm{Sb}_{1.5} \mathrm{Te}_{3}$ films onto $\mathrm{Si} / \mathrm{SiO}_{2}$ wafers. Mobilities observed in the films were approximately 5 times lower than those observed in single crystals. This disappointing reduction in mobility was attributed to the scattering of charge carriers by imperfections, though the work appears to be inconclusive. The best work on sputtered films of $(\mathrm{Bi}, \mathrm{Sb})_{2}(\mathrm{Te}, \mathrm{Se})_{3}$ was recently published by Noro, Sato, and Kagechika [1993]. The Seebeck coefficient $(\alpha)$ and electrical conductivity $(\sigma)$ were measured at ambient temperature. Measurements were made for films prepared with different substrate and annealing temperatures. These investigators conclude that potentially, sputtered polycrystalline films can have the same thermoelectric performance as bulk single crystals.

One-Dimensional Quantum Wells. Theoretical predictions indicate that an appropriately engineered one-dimensional quantum well might also have an enhanced dimensionless figure-ofmerit [Hicks and Dresselhaus, 1993]. Some believe that carbon nanotubes might behave like like such quantum wells. To investigate this possibility, investigators at Lawrence Berkeley National Laboratory (another site operated by the University of California) and Rice University have prepared films osf single-walled carbon nanotubes [Hone et al., 1998]. Unfortunately, the $\mathrm{ZT}_{3 \mathrm{D}}$ observed with these films does not appear to be greater than the values achievable with more conventional materials. 
White Paper for U.S. Army Rapid Equipping Force - Joseph C. Farmer November $22^{\text {nd }} 2007$

Table 1 - Characteristics of Candidate Thermoelectric Materials (some ZT's estimated)

Family Type \& Compound

$\mathrm{T}(\mathrm{K}) \quad \mathrm{ZT} \quad$ Source

Tellurides

$\begin{array}{ll}\mathrm{p} & \mathrm{Bi}_{0.5} \mathrm{Sb}_{1.5} \mathrm{Te}_{3} \\ \mathrm{n} & \mathrm{Bi}_{2} \mathrm{Te}_{1.4} \mathrm{Se}_{0.6} \\ \mathrm{p} & \mathrm{PbTe} \\ \mathrm{n} & \mathrm{PbTe}^{\mathrm{n}} \\ \mathrm{n} & \mathrm{Pb}_{0.75} \mathrm{Sn}_{0.25} \mathrm{Te} \\ \mathrm{p} & \left(\mathrm{GeTe}_{0.95}\left(\mathrm{Bi}_{2} \mathrm{Te}_{3}\right)_{0.05}\right. \\ \mathrm{p} & \mathrm{AgSbTe}_{2} \\ \mathrm{p} & {\mathrm{Amorphous} \mathrm{As}_{2} \mathrm{Te}_{3}}\end{array}$

$\begin{array}{lll}300 & 0.98 & \text { Rosi, } 1968\end{array}$

$300 \quad 0.72 \quad$ Rosi, 1968

$\begin{array}{lll}600 & 1.05 & \text { Rosi, } 1968\end{array}$

$600 \quad 1.05 \quad$ Rosi, 1968

$900 \quad 1.44 \quad$ Wood, 1988

$\begin{array}{lll}750 & 1.28 & \text { Wood, } 1988\end{array}$

$\begin{array}{lll}650 & 1.17 & \text { Wood, } 1988\end{array}$

$\begin{array}{lll}773 & 0.33 & \text { Mott, } 1979\end{array}$

Si-Ge Alloys

$\begin{array}{ll}\mathrm{p} & \mathrm{Si}_{70} \mathrm{Ge}_{30} \\ \mathrm{n} & \mathrm{Si}_{70} \mathrm{Ge}_{30}\end{array}$

$1000 \quad 1.00 \quad$ Rosi, 1968

$1000 \quad 1.10 \quad$ Rosi, 1968

Silicides

$\begin{array}{ll}\mathrm{n} & \mathrm{FeSi}_{2}+3 \% \mathrm{Co} \\ \mathrm{p} & \mathrm{Ru}_{2} \mathrm{Si}_{3} \\ \mathrm{n} & \mathrm{Ru}_{2} \mathrm{Si}_{3}\end{array}$

$1000 \quad 0.08 \quad$ Matsubari, 1992

$700 \quad 0.002$ Ohta, 1992

$500 \quad 0.006$ Ohta, 1992

Sulfides

$\begin{array}{ll}\mathrm{p} & \mathrm{Ce}_{3-\mathrm{x}} \mathrm{S}_{4}(0.00<\mathrm{x}<0.33) \\ \mathrm{p} & \mathrm{Ce}_{3-\mathrm{x}} \mathrm{S}_{4}(0.30<\mathrm{x}<0.33) \\ \mathrm{n} & \mathrm{LaS}_{1.445} \\ \mathrm{p} & \mathrm{US}\end{array}$

1000

0.43

Cutler, 1964

$1000 \quad 1.03 \quad$ Cutler, 1964

$1000 \quad 1.53 \quad$ Kamarzin, 1981

$573 \quad 0.06 \quad$ Gmelin

Antimonides

$\mathrm{p} \quad \mathrm{IrSb}_{3}$

$773 \quad 0.50 \quad$ Caillat, 1992

HTSCs

p $\quad \mathrm{YBa}_{2} \mathrm{Cu}_{3} \mathrm{O}_{6.3}$

$300 \quad 0.06 \quad$ Macklin, 1990

Polymers

p Polyacetylene $+\mathrm{FeCl}_{3}$

300

Superlattice

$\mathrm{PbTe} / \mathrm{Te}$

1.9 Harman, 1998 


\section{Lithium Ion Systems}

The secondary battery system proposed for this application will be constructed from individual polymer-gel lithium-ion batteries. The best commercially available, polymer-gel lithium ion battery now has a specific energy of $\sim 180 \mathrm{Wh} / \mathrm{kg}$, an energy density of $\sim 350 \mathrm{Wh} / \mathrm{L}$, and a reasonably good rate capability, allowing discharge at $\mathrm{C} / 2$. Unlike the silver-zinc systems which have been used historically for underwater applications, lithium-ion systems have much longer cycle life (by orders-of-magnitude), providing dramatic cost advantages for large-scale systems. Lead acid systems have also been used, but require large volumes soluble lead, corrosive sulfuric acid, and have the potential for substantial gas-generation. In the case of lithium-ion batteries, chemistry is usually based on transition-metal oxide intercalation cathodes, graphitic intercalation anodes, and aprotic solvents with lithium salts. Solid-state and polymer electrolyte systems are also possible, but usually are limited by poor rate capability. In the case of conventional secondary battery systems, high capacity will be achieved with series-parallel networks of smaller cells, a strategy which has already been demonstrated. Such integrated networks require charging systems that enable cell balancing, as well as the removal of failed cells from the network.

A typical polymer gel lithium ion battery is shown in Figure 7. Both liquid prismatic and polymer gel cells have been incorporated into large high-capacity power packs, like the one shown in Figure 8, and used to power the mobile Heat Capacity Laser (HCL) at LLLNL. Such high capacity systems have state-of-the-art computerized charge and discharge control, which includes graphical user interfaces, sensing for monitoring the health of individual cells, and charge balancing networks
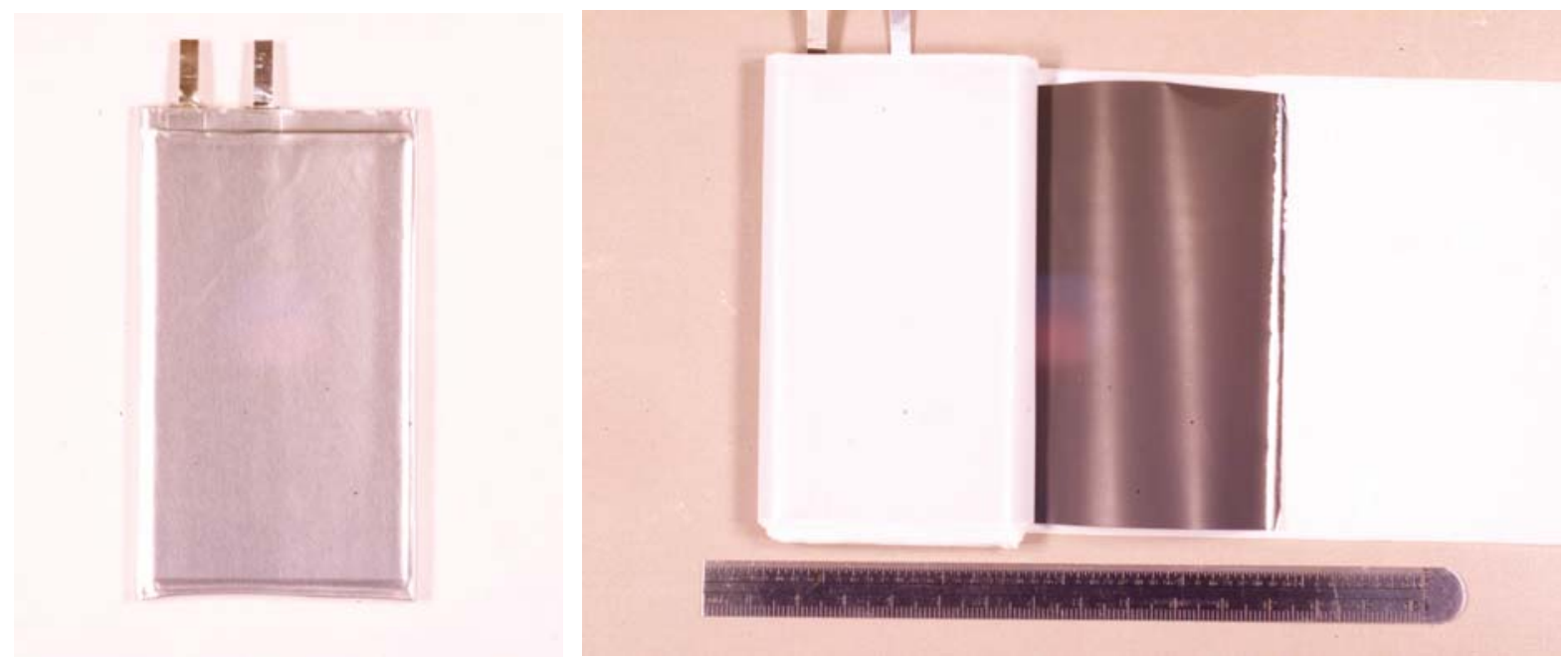

Figure 7 - Conventional polymer gel lithium ion battery used in laptops and wireless devices, such as cellular telephones. 


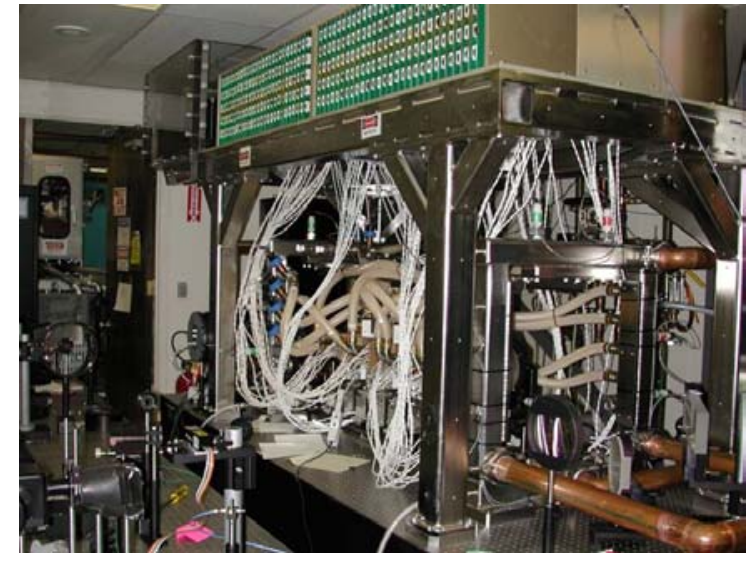

Diode-Pumped HCL

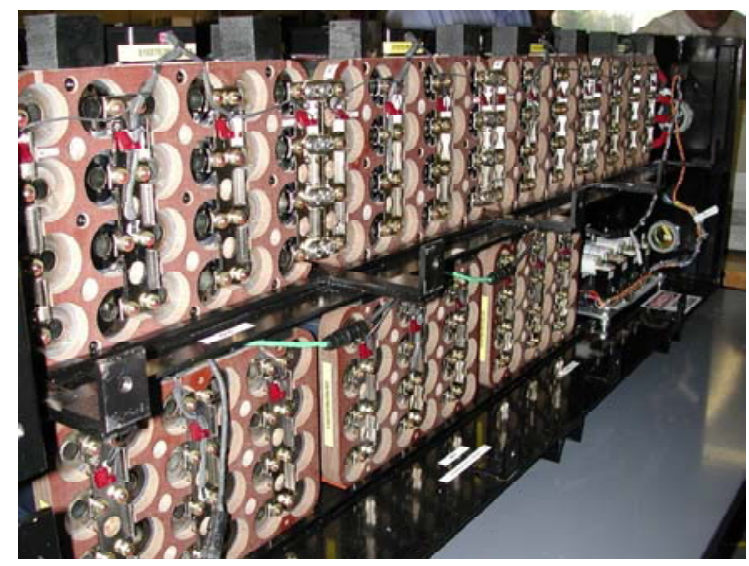

1.5 MW Lithium Ion Battery

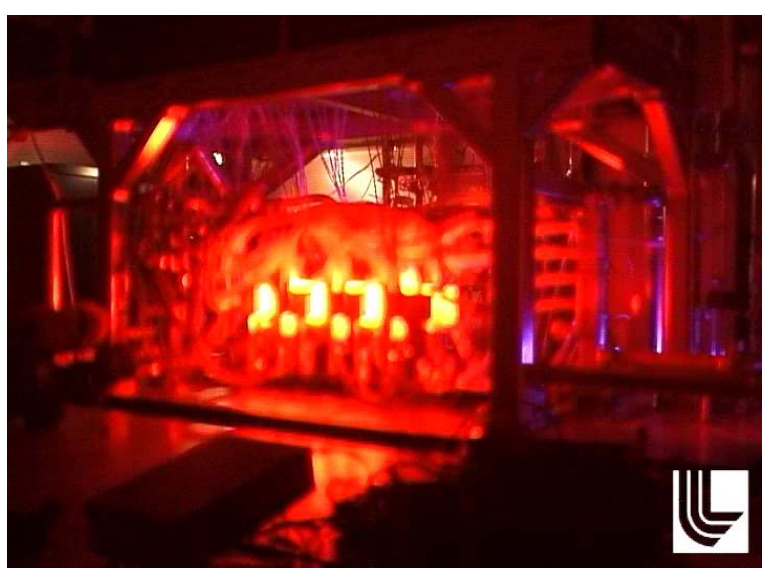

Firing HCL -30 kW Pulse

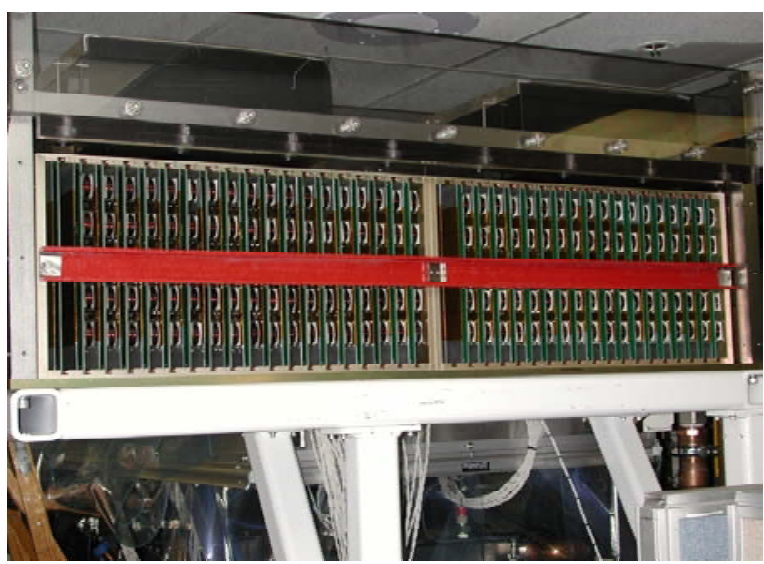

Laser Pulse Forming Network

Figure 8 -Mobile Heat Capacity Laser (HCL) with high-capacity lithium-ion battery packs.

New solid-state fast ion conductors could used to enhance the performance of rechargeable solidstate lithium-ion batteries, through the elimination of potentially volatile and combustible electrolyte solvents. A high-conductivity, solid-state, composite electrolyte system could eventually be developed that is mechanically robust and thermo-chemically stability. This composite material will involve new organic solid-polymer electrolytes, and will the construction of cells with an electrolyte/separator thickness less than 25 microns, and electrolyte conductivity greater than $10 \mathrm{mS} / \mathrm{cm}$ at $0^{\circ} \mathrm{C}$. The best current materials have an order-of-magnitude lower conductivity, and lack stability in contact with the cathode, which exists at a high anodic (oxidizing) potential. Such new materials will enable the construction of inherently safe, highrate, high-capacity solid-state lithium cells. Substitution, inorganic constituents, and radiation cross-linking would be employed as required.

New anode materials could be developed to provide a specific capacity of $750 \mathrm{Ah} / \mathrm{kg}$, and a capacity density of $1700 \mathrm{Ah} / \mathrm{L}$. Intercalated graphite $\left(\mathrm{LiC}_{6}\right)$ is the industry standard for the active material used to fabricate anodes in polymer-gel lithium-ion batteries, and has a theoretical specific capacity of $372 \mathrm{Ah} / \mathrm{kg}$, and a theoretical capacity density of $837 \mathrm{Ah} / \mathrm{L}$ (the practical 
values are lower). The target materials to be developed as part of this program will therefore exceed the theoretical values for the industry standard materials by a factor of two $(\times 2)$.

Similarly, new cathode material will be developed to provide a specific capacity of $550 \mathrm{Ah} / \mathrm{kg}$, and a capacity density of $2040 \mathrm{Ah} / \mathrm{L}$. Lithium cobalt oxide $\left(\mathrm{LiCoO}_{2}\right)$ is the industry standard for the active material used to fabricate cathodes in polymer-gel lithium-ion batteries, and has a theoretical specific capacity of $274 \mathrm{Ah} / \mathrm{kg}$, and a theoretical capacity density of $1017 \mathrm{Ah} / \mathrm{L}$ (the practical values are lower). The target materials to be developed as part of this program will therefore exceed the theoretical values for the industry standard materials by a factor of two $(\times 2)$.

Ultimately, a new inherently safe, high-energy, high-rate solid-state rechargeable battery could be fabricated as a composite solid-state material. Advancements made through materials science will enable the construction of a solid-state rechargeable battery will have a specific capacity of $360 \mathrm{Ah} / \mathrm{kg}$ and a volumetric capacity density of $700 \mathrm{Ah} / \mathrm{L}$. A typical solid polymer electrolyte (SPE) cell has a specific energy of only $\sim 100 \mathrm{Wh} / \mathrm{kg}$, and an energy density of only $\sim 80 \mathrm{Wh} / \mathrm{L}$ (though some higher values have been sporadically reported). This achievement would provide the first robust solid-state rechargeable battery for underwater applications, and would double the mission duration possible with the best commercially available polymer-gel technology. It would increase the duration possible with existing SPE-type cells by an order-of-magnitude $(\times 10)$. Such and advancement in lithium ion battery technology could provide higher specific energy, higher volumetric energy density, higher rate capability, and safer and more reliable operation.

\section{Liquid Redox Batteries}

Other promising systems for high-capacity stationary storage batteries include various liquid redox couples, with oxidants and reductants which exist in the liquid phase at ambient temperature and pressure, and can be stored in tanks. Scaling for capacity for such systems is relatively simple, in that it requires larger tanks. Rate capability is determined by the masstransport limited current density, which can be controlled through good cell engineering, with flow channels that promote good mass-transfer, and high efficiency electrode-separator stacks. The system must be designed with good heat transfer in mind, to prevent overheating and thermal runaway. 